\title{
THE EXISTENCE OF EIGENVALUES FOR INTEGRAL OPERATORS $\left({ }^{1}\right)$ \\ BY \\ SAMUEL KARLIN
}

In the following paper we establish conditions for the existence of an infinite, simple point spectrum (and properties of the corresponding eigenfunctions $\left\{\phi_{n}\right\}$ ) for the integral operator

$$
(T \phi)(x)=\int_{a}^{b} K(x, s) \phi(s) d s, \quad a \leqq x \leqq b .
$$

Throughout $K(x, s)$ is assumed to be real valued and continuous for $\Delta \times \Delta(\Delta=[a, b]$ is a finite closed interval of the real line). For definiteness we fix the domain $\mathscr{D}(T)$ of the integral operator (1) as the Hilbert space $L_{2}(\Delta)$; it should be emphasized at this point that $K(x, s)$ is not necessarily a symmetric kernel. It will be clear from the subsequent analysis that the nature of the spectral set of $T$ is unaltered for any of the alternative specifications $\mathscr{D}(T)=L_{p}(\Delta), 1 \leqq p \leqq \infty$.

It is well known that $T$ is completely continuous. Therefore, the eigenvalues $\Lambda=\left\{\lambda_{0}, \lambda_{1}, \lambda_{2}, \cdots\right\}$ form a discrete set which may be infinite, finite or empty. Each eigenvalue is of finite algebraic and geometric multiplicity and 0 is the only limit point of $\left\{\lambda_{i}\right\}$ if $\Lambda$ is not finite. Finally, the spectrum of the transformation $T$, apart from point spectrum $\Lambda$, can contain only the origin. Let $r(T)$ denote the spectral radius of $T$, i.e.,

$$
r(T)=\max _{\lambda_{i} \in \Lambda}\left|\lambda_{i}\right|
$$

For $\lambda>r(T)$ the Neumann expansion applies (convergence is understood in the sense of the operator norm):

$$
\left(I-\frac{T}{\lambda}\right)^{-1}=\sum_{m=0}^{\infty} \frac{T^{m}}{\lambda^{m}} .
$$

The iterated operator $T^{m}$ is associated with the iterated kernel $K^{(n)}(x, s)$ defined by

$$
K^{(n)}(x, s)=\int_{\Delta} \cdots \int_{\Delta} K\left(x, s_{1}\right) K\left(s_{1}, s_{2}\right) \cdots K\left(s_{n-1}, s\right) d s_{1} \cdots d s_{n-1} .
$$

Specifically,

Received by the editors May 22, 1963.

(1) In honor of my friend and colleague Professor C. Loewner on his 70th Birthday. 


$$
T^{n} \phi(x)=\int_{a}^{b} K^{(n)}(x, s) \phi(s) d s \quad(a \leqq x \leqq b) .
$$

It is also familiar that the conjugate operator

$$
T^{*} \psi(s)=\int_{a}^{b} K(x, s) \psi(x) d s
$$

possesses the same spectrum as $T$.

The existence of eigenvalues for (1) is known in two cases. The first, that of symmetric kernels, is classic. For example if $K(x, s)=K(s, x)$ and $K$ is positive definite, i.e.,

$$
\begin{aligned}
& K\left(\begin{array}{c}
x_{1}, x_{2}, \cdots, x_{n} \\
x_{1}, x_{2}, \cdots, x_{n}
\end{array}\right)=\operatorname{det}\left\|K\left(x_{i}, x_{j}\right)\right\|>0 \\
& a \leqq x_{1}<x_{2}<\cdots<x_{n} \leqq b, n=1,2,3, \cdots,
\end{aligned}
$$

then $T$ possesses a countable set of positive eigenvalues $\Lambda=\left\{\lambda_{i}\right\}_{i=0}^{\infty}$ and a corresponding complete orthonormal set of eigenfunctions $\left(\phi_{i}(x)\right\}_{i=0}^{\infty}$.

In the case in which the kernel $K(x, s)$ is symmetric and, moreover, satisfies not only (7), but the more extensive system of inequalities

$$
\begin{gathered}
K\left(\begin{array}{l}
x_{1}, x_{2}, \cdots, x_{n} \\
s_{1}, s_{2}, \cdots, s_{n}
\end{array}\right)=\operatorname{det}\left\|K\left(x_{i}, s_{j}\right)\right\| \geqq 0, \\
a \leqq x_{1}<x_{2}<\cdots<x_{n} \leqq b ; a \leqq s_{1}<\cdots<s_{n} \leqq b ; n=1,2,3, \cdots,
\end{gathered}
$$

it is proved by Gantmacher and Krein [1] that the eigenfunctions $\left\{\phi_{i}\right\}_{i=0}^{\infty}$ enjoy elaborate oscillation properties. Specifically, each $\lambda_{i}$ is of multiplicity 1 and the functions $\phi_{n}(x)$ obey the inequalities

$$
\Phi\left(\begin{array}{cc}
0, & 1, \cdots, m-1 \\
x_{1}, x_{2}, \cdots, \quad x_{m}
\end{array}\right)=\operatorname{det}\left(\left\|\phi_{i}\left(x_{j}\right)\right\|_{i=0, j=1}^{m-1, m}\right) \neq 0
$$

for all choices $a \leqq x_{1}<x_{2}<x_{3}<\cdots<x_{m} \leqq b ; m=1,2,3, \cdots$. This inequality implies that $\phi_{i}(x)$ has precisely $i$ zeros and zeros of successive eigenfunctions strictly interlace. Once a determination of signs is made as to which sign should be taken in $\pm \phi_{n}(x)$ then the sign in (9) can be made explicit. In fact, under the stipulation $\phi_{n}(a)>0$ (we remark it can be proven that $\phi_{n}(a) \neq 0$ for all $n$ ), then (9) can be written more precisely in the form

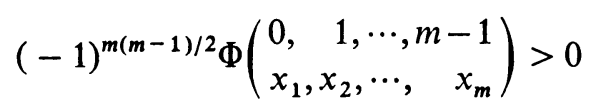

The second class of operators (not necessarily self-adjoint) for which the isextence of some eigenvalues and eigenfunctions is demonstrated are those induced by non-negative kernels. The familiar Frobenius-Perron-Jentzsch theorem (see Theorem 1 below) asserts that if $K(x, s)$ is strictly positive on $\Delta \times \Delta$ then the spectral radius $r(T)>0$ is an eigenvalue whose eigenmanifold possesses a 
strictly positive function. Observe that this proposition merely affirms the existence of only one eigenvalue contrasting to the symmetric case in which, if $T$ is not finite dimensional, the existence of a countable spectral set is guaranteed. The strictness postulate is essential in the sense that if $K(x, s)$ is assumed only nonnegative, then as shown by the example of the Volterra kernel

$$
(V f)(x)=\int_{a}^{x} f(s) d s=\int_{a}^{b} M(x, s) f(s) d s
$$

whose associated kernel $M(x, s)=0$ or 1 according as $x<s$ or $s \leqq x$ shows that no eigenvalues need exist. The fact that $M(x, s)$ is not continuous is not relevant to the example since the same result obtains if $M(x, s)$ is continuous but with support confined to the region $s \leqq x$.

In this paper we obtain an extension of (10) to the nonsymmetric operator (1) by postulating a condition slightly stronger than (8). In order to formulate our results, we need to introduce some new concepts and discuss their relevance. A kernel $K(x, s) \in C^{\infty}$ (i.e., infinitely continuously differentiable) is said to be extended totally positive (abbreviated ETP) if

$$
\operatorname{det}\left(\frac{\partial^{i+j} K(x, s)}{\partial x^{i} \partial s^{j}}\right)_{i, j=0}^{n}>0, \quad n=0,1,2, \cdots ; x, s \in \Delta \times \Delta .
$$

It is not difficult to prove that (12) implies (8) with strict inequality [3].

At this point it is convenient to fix some notation and terminology. Let

$$
\Delta_{p}=\left\{\bar{x}=\left(x_{1}, x_{2}, \cdots, x_{p}\right) \mid a<x_{1}<x_{2}<\cdots<x_{p}<b\right\}
$$

denote the open simplex and

$$
\bar{\Delta}_{p}=\left\{\bar{x}=\left(x_{1}, x_{2}, \cdots, x_{p}\right) \mid a \leqq x_{1} \leqq x_{2} \leqq \cdots \leqq x_{p} \leqq b\right\}
$$

its closure in $E^{p}$. Notice that by our notation $\Delta=\bar{\Delta}_{1}$. Sometimes it is useful to work with

$$
\tilde{\Delta}_{p}=\left\{\bar{x}=\left(x_{1}, x_{2}, \cdots, x_{p}\right) \mid a \leqq x_{1}<x_{2}<\cdots<x_{p} \leqq b\right\} .
$$

The kernel

$$
K_{[p]}(\bar{x}, \bar{s})=K\left(\begin{array}{c}
x_{1}, x_{2}, \cdots, x_{p} \\
s_{1}, s_{2}, \cdots, s_{p}
\end{array}\right), \quad \begin{aligned}
& \bar{x}=\left(x_{1}, x_{2}, \cdots, x_{p}\right) \in \Delta_{p}, \\
& \bar{s}=\left(s_{1}, s_{2}, \cdots, s_{p}\right) \in \Delta_{p},
\end{aligned}
$$

s defined on $\Delta_{p} \times \Delta_{p}$ and is called the compound kernel of order $p$ induced by $K(x, s)$. The operator $T_{[p]}$ is defined on $L^{2}\left(\bar{\Delta}_{p}\right)$ as in (1) by the kernel (16) where $K_{[p]}(\bar{x}, \bar{s})$ is extended to the boundary of $\bar{\Delta}_{p}$ by continuity.

A compound kernel obeying the conditions (8) with strict inequality on $\Delta_{p} \times \Delta_{p}$ describes a strictly positive function there which, however, necessarily vanishes on at least part of the boundary (e.g., when $x_{i}=x_{i+1}$ for some $i$ ).

If $K(x, s)$ is of class $C^{\infty}$, there exists a useful way of associating with $K$ a kernel 
defined on $\bar{\Delta}_{p} \times \bar{\Delta}_{p}$ which may retain strict positivity even on this extended domain.

Let

$$
\begin{aligned}
& u_{1}(x) \equiv 1, \quad x \in \Delta, \\
& u_{p}(\bar{x})=\prod_{1 \leqq i<j \leqq p}\left(x_{j}-x_{i}\right) \bar{x}=\left(x_{1}, x_{2}, \cdots, x_{p}\right) \in \Delta_{p} \quad(p \geqq 2) .
\end{aligned}
$$

By suitably invoking the mean value theorem it is easy to see that

$$
\frac{K(\bar{x}, \bar{s})}{u_{p}(\bar{x}) u_{p}(\bar{s})}
$$

can be extended continuously to $\bar{\Delta}_{p} \times \bar{\Delta}(p=1,2, \cdots)$ (see $\left.[3 ; 4]\right)$.

Definition. A kernel $K(x, s) \in C^{\infty}$ is said to be $\operatorname{ETP}(x)$ if

$$
L_{p}(\bar{x}, \bar{s})=\frac{K(\bar{x}, \bar{s})}{u_{p}(\bar{x})}>0 \text { for } \bar{x} \in \bar{\Delta}_{p}, \bar{s} \in \Delta_{p}
$$

$\operatorname{ETP}(s)$ if

$$
\tilde{L}_{p}(\bar{x}, \bar{s})=\frac{K(\bar{x}, \bar{s})}{u_{p}(\bar{s})}>0 \text { for } \bar{x} \in \Delta_{p}, \bar{s} \in \bar{\Delta}_{p}
$$

and ETP (with no reference to $\bar{x}$ or $\bar{s}$ ) if

$$
M_{[p]}(\bar{x}, \bar{s})=\frac{K(\bar{x}, \bar{s})}{u_{p}(\bar{x}) u_{p}(\bar{s})}>0 \text { for } \quad x \in \bar{\Delta}_{p}, s \in \bar{\Delta}_{p} \quad(p=1,2,3, \cdots) .
$$

We prove in [4] the interesting fact that the definitions (12) and (21) are equivalent. Its method consists of a judicious exploitation of the mean value property (cf. [3]). It is also shown that if (8) is non-negative and $K(x, s) \in C^{\infty}$ then the functions $L_{[p]}(\bar{x}, \bar{s}), \tilde{L}_{p}(\bar{x}, \bar{s})$ and $M_{[p]}(\bar{x}, \bar{s})$ are each non-negative (not necessarily positive) on their domains of definitions.

One more bit of notation. If $f_{1}, f_{2}, \cdots$ denote a sequence of $C^{\infty}$ functions defined on $[a, b]$, we extend

$$
F\left(\begin{array}{c}
1,2, \cdots, p \\
x_{1}, x_{2}, \cdots, x_{p}
\end{array}\right)=\operatorname{det}\left\|f_{i}\left(x_{j}\right)\right\|, \quad \bar{x} \in \Delta_{p},
$$

to $\bar{x} \in \bar{\Delta}_{p}$ as the continuous extension of

$$
F^{*}\left(\begin{array}{c}
1,2, \cdots, p \\
x_{1}, x_{2}, \cdots, x_{p}
\end{array}\right)=\frac{F\left(\begin{array}{c}
1,2, \cdots, p \\
x_{1}, x_{2}, \cdots, x_{p}
\end{array}\right)}{u_{p}(\bar{x})}
$$

The * symbol will always occur with the interpretation as indicated above.

With these definitions in hand we state the principal theorem of this paper. (Theorems 1 and 2 of $\S 2$ serve primarily as auxiliarly assertions.) 
THEOREM 3. Let $K(x, y)$ be of class $C^{\infty}$ and ETP on $[a, b]$. Then the operator $T$ possesses a countable set of simple positive eigenvalues

$$
\lambda_{0}>\lambda_{1}>\lambda_{2}>\cdots>\lambda_{n}>\cdots
$$

decreasing to zero. There exists no other nonzero spectrum apart from the origin. Let $\phi_{0}(x), \phi_{1}(x), \phi_{2}(x), \cdots$ denote the corresponding eigenfunctions (each uniquely determined except for a multiplicative factor). Then

$$
\operatorname{sign} \varepsilon_{r} \Phi^{*}\left(\begin{array}{c}
0,1, \cdots, r \\
x_{1}, x_{2}, \cdots, x_{r}
\end{array}\right)>0 \quad\left(\bar{x} \in \bar{\Delta}_{r}\right)
$$

where $\varepsilon_{r}$ is appropriately +1 or -1 .

We will discuss in $\S 4$ the relevance of the inequality (24) with regard to oscillation properties of the eigenfunctions.

A general class of kernels which satisfy the conditions of this theorem have the form

$$
K(x, s)=\int_{0}^{\infty} e^{u(x) \alpha(t)} e^{v(s) \beta(t)} d \sigma(t), \quad a \leqq x, s \leqq b,
$$

where $u, v$ are of class $C^{\infty}, u^{\prime}(x) \cdot v^{\prime}(x)>0, \alpha(x)$ and $\beta(x)$ are strictly increasing functions and $d \sigma$ is a sigma finite positive measure with an infinite number of points of increase. The integral is assumed to converge absolutely for $a \leqq x$, $s \leqq b$. A special case of $(25)$ is

$$
K(x, s)=\sum_{n=0}^{\infty} a_{n}[u(x)]^{n}[v(s)]^{n}
$$

$\left(a_{n} \geqq 0\right.$ for all $n$ and $>0$ for infinitely many $n$ ).

The verification that (25) is ETP is done in [3].

The result of Theorem 3 can be generalized in many ways. For example, one can consider $T_{\mu} f(x)=\int_{a}^{b} K(x, s) f(s) d \mu(s)$ where $d \mu$ is a suitable measure on $[a, b]$ subject to certain requirements.

Secondly, it is clear that since the eigenfunctions $T$ and $T^{k}$ are identical and the eigenvalues are simply related $\left(\lambda\right.$ to $\left.\lambda^{k}\right)$, it suffices that our analysis apply to some iterate $T^{k_{0}}$, rather than to $T$ itself.

We shall indicate some of these extensions in $\$ 4$ as well as a statement of other results pertaining to the nature of the eigenfunctions $\left\{\phi_{n}\right\}_{n=0}^{\infty}$. We intend to publish elsewhere these more refined assertions concerning the structure of the eigenfunctions $\left\{\phi_{n}\right\}$.

In $\$ 2$ we develop several variations on the Frobenius-Perron-Jentzsch theorem which will be required in the detailed proof of Theorem 3 .

2. The Frobenius-Perron-Jentzsch theorem and some ramifications. We preface our study of the spectral characteristics of (1) with a discussion of two theorems 
essential to the proofs of our main result. Throughout this section (unless stated explicitly to the contrary), we will assume $K(x, s)$ is continuous on the closed rectangle $S \times S$ where $S$ is a compact subset of some Euclidean space such that $S=\overline{S^{0}}\left(S^{0}=\right.$ interior of $\left.S\right)$.

The first theorem we need is a form of the Frobenius-Perron-Jentzsch theorem concerning the largest eigenvalue of non-negative matrices or of integral operators with non-negative kernel.

THEOREM 1. If $K(x, y) \geqq 0$ on $S \times S$ and $r(T)=$ spectral radius of $T$ is positive, then

(i) $r(T)$ is an eigenvalue of $T$.

(ii) There exists a non-negative eigenfunction corresponding to the eigenvalue $r(T)$.

The proof of Theorem 1 can be found in numerous sources (see $[5 ; 6 ; 7]$, as typical).

The subsidiary hypothesis that $r(T)>0$ rules out the possibility of $T$ being quasi nilpotent as in the case of the Volterra operator (11).

A useful characterization of the spectral radius as introduced in [7] will be helpful for our present purposes.

Let $\psi^{*}(s)$ be a fixed continuous everywhere positive function on $S$. A real number $\gamma$ is said to be admissible if there exists a non-null continuous function $\phi(s) \geqq 0(s \in S)$ such that

$$
(T \phi)(x) \geqq \gamma \phi(x), \quad x \in S,
$$

and $\phi$ is normalized so that

$$
\int_{a}^{b} \phi(s) \psi^{*}(s) d s=1 .
$$

We also write (26) compactly as $T \phi \geqq \gamma \phi$. Let $\Gamma=\{\gamma \mid \gamma$ admissible $\}$. Clearly $0 \in \Gamma$ and it is easy to verify that $\Gamma$ is bounded. Let

$$
\gamma_{0}=\sup _{\gamma \in \Gamma} \gamma .
$$

Complete continuity of $T$ implies $\gamma_{0} \in \Gamma$ (cf. [7]), while positivity of $K(x, s)$ implies

$$
T^{n} \phi_{0} \geqq \gamma_{0}^{n} \phi_{0}
$$

as a consequence of $T \phi_{0} \geqq \gamma_{0} \phi_{0}$.

Since $r(T)=\lim _{n \rightarrow \infty}\left\|T^{n}\right\|^{1 / n}$, we see that whenever $T$ is positive then $r(T) \geqq \gamma_{0}$. If it is known that $r(T)>0$ then Theorem 1 tells us that $r(T)$ itself is admissible and so $r(T)=\gamma_{0}$. This is the desired characterization of $r(T)$ by (27) as a supremum. 
In particular, if $K(x, s)$ is strictly positive on $S \times S$ then plainly $\Gamma$ includes positive numbers and so $r(T)=\gamma_{0}$. Actually, for $K(x, s)>0$ on $S \times S$ the conclusions of Theorem 2 are valid.

THeORem 2. Suppose $K(x, s)>0$ for each $x \in S$ and all $s \in S^{0}=$ interior of $S$ (remember that $S=\overline{S^{0}}$ ). Then

(i) $r(T)=\gamma_{0}>0$.

(ii) $\gamma_{0}$ is a simple eigenvalue possessing a strictly positive eigenfunction $\phi_{0}(x)$; the characteristic manifold of $\gamma_{0}$ is one-dimensional and $\gamma_{0}$ is a simple pole of the resolvent $R(\lambda, T)=(\lambda I-T)^{-1}$.

(iii) If $\lambda$ is an eigenvalue of $T, \lambda \neq \gamma_{0}$, then $|\lambda|<\gamma_{0}$.

(iv) $\left(T / \gamma_{0}\right)^{n}$ converges to the one-dimensional projection operator on the eigenmanifold $\mathscr{M}\left(\gamma_{0}\right)=\left\{\phi \mid\left(T-\gamma_{0} I\right) \phi=0\right\}$. Moreover, $L_{2}(S)$ decomposes into the direct sum $\mathscr{M}\left(\gamma_{0}\right) \oplus \mathscr{U}\left(\gamma_{0}\right)$ where $\mathscr{U}\left(\gamma_{0}\right)$ is a complementary invariant subspace on which $T$ has spectral radius less than $\gamma_{0}$.

We refer the reader to [5] or [7] for details of the proof. In connection with (iv) see also [8].

Theorem 2, in the form in which we have just stated it, is not adequate for our purposes. The hypothesis that $K$ be strictly positive on $S \times S^{0}$ is more than will be fulfilled in our applications. The vanishing of $K(x, s)$ for $x \in \mathrm{Bd} S$ cannot be avoided in some circumstances.

For example, suppose we assume that $K(x, s)$ is ETP on $\Delta \times \Delta$. In particular $K(x, s)>0$ for $\Delta \times \Delta$ and so Theorem 2 is applicable. On the other hand the compound kernel $K_{[p]}(\bar{x}, \bar{s})$ is positive only for $\Delta_{p} \times \Delta_{p}$ and in fact vanishes on the boundary of $\bar{\Delta}_{p} \times \bar{\Delta}_{p}$. This necessitates investigating the validity of Theorem 2 in such a situation.

It is, therefore, of some intrinsic interest, as well as crucial for our applications below, that the following form of Theorem 2 does hold:

THEOREM 2'. Assume $K(x, s)$ is positive on $S^{0} \times S^{0}$, while $K(x, s)$ may vanish when $x$ and/or $s$ lie on the boundary of $S$. However, assume there exists a continuous function $u(x)$, positive on $S^{0}$ such that

$$
G(x, s)=\frac{K(x, s)}{u(x)}
$$

is continuous on $S \times S$ and positive on $S \times S^{0}$ (i.e., $G(x, s)$ can be extended to the boundary in $x$ so as to remain positive).

Then conclusions (i)-(iv) of Theorem 2 remain valid except $\phi_{0}(x) / u(x)$ is asserted to be positive on $S$ rather than $\phi_{0}(x)$ itself.

Proof. If $\phi(x)$ is positive on $S^{0}$ and continuous on $S$, then so is $\tilde{\phi}=T \phi$, and we have that 


$$
\frac{\tilde{\phi}(x)}{u(x)}=\frac{(T \phi)(x)}{u(x)}
$$

is continuous and positive in $S$.

Now for some $\alpha>0$

$$
\frac{T \tilde{\phi}(x)}{u(x)} \geqq \alpha \frac{\tilde{\phi}(x)}{u(x)} \text { or } T \tilde{\phi}(x) \geqq \alpha \tilde{\phi}(x) \text {. }
$$

This shows that $\gamma_{0}>0$ and so $r(T)=\gamma_{0}>0$ and (i) is correct.

Notice that if $\phi_{0} \geqq 0$ is an eigenfunction of $\gamma_{0}$ then we have that $\phi_{0}(x) / u(x)$ is positive on $S$. If $\phi(x)$ is any other real eigenfunction associated with $\gamma_{0}$, then we can by a suitable choice of $\delta$ ensure that

$$
\frac{\phi^{*}(x)}{u(x)}=\frac{\phi(x)}{u(x)}+\delta \frac{\phi_{0}(x)}{u(x)}
$$

be zero somewhere in $S$ and otherwise non-negative. But $T \phi^{*}=\gamma_{0} \phi^{*}$ entails that $\phi^{*}(x) / u(x)$ is positive on $S$ or identically zero. By the constructions, only the second alternative can hold and therefore $\phi$ is a multiple of $\phi_{0}$. This shows that the eigenmanifold of $\gamma_{0}$ is one-dimensional.

Next, let $\psi_{0} \geqq 0$ be the unique eigenfunction of $T^{*}$ for $\gamma_{0}$. It follows readily that $\psi_{0}(s)>0, s \in S^{0}$. Using this fact, the proof of the assertion that the algebraic multiplicity of $\gamma_{0}$ is 1 is now standard and will be omitted (see [7, pp. 921922]).

We claim that if $T \phi \geqq \gamma_{0} \phi$ and $\phi \geqq 0(\phi$ continuous and $\not \equiv 0)$, actually equality holds. We exhibit the usual proof (cf. [7]). If inequality holds for some $x$, then since $\psi_{0}(s)>0, s \in S^{0}$, we have

$$
\left(T \phi-\gamma_{0} \phi, \psi_{0}\right)>0 \text {. }
$$

(Here, $(v, w)$ denotes the inner product of the functions $v$ and $w$.) But

$$
0<\left(\left(T-\gamma_{0} I\right) \phi, \psi_{0}\right)=\left(\phi,\left(T^{*}-\gamma_{0} I\right) \psi_{0}\right)=0,
$$

an absurdity. This contradiction proves the assertion to the effect that if $T \phi \geqq \gamma_{0} \phi, \phi \neq \equiv$, then $T \phi \equiv \gamma_{0} \phi$.

We turn now to (iii). Consider the relation

$$
T \phi^{*}=\lambda \phi^{*}, \quad|\lambda|=\gamma_{0} \neq \lambda,
$$

with $\phi^{*}$ non-null. Taking absolute values, we obtain

$$
T\left(\left|\phi^{*}\right|\right) \geqq \gamma_{0}\left|\phi^{*}\right|=\left|T \phi^{*}\right|
$$

(Here, $\left|\phi^{*}\right|$ denotes the function $\left|\phi^{*}(x)\right|$.) As we have just proved, equality must then hold in (30). Written out this asserts 


$$
\left|\int_{s} K(x, s) \phi^{*}(s) d s\right|=\int_{s} K(x, s)\left|\phi^{*}(s)\right| d s
$$

for all $x$. This requires that the values $K(x, s) \phi^{*}(s)$ for all $s$ lie along a single ray in the complex plane. But $K(x, s)>0$ for $x, s \in S^{0} \times S^{0}$ and therefore $\phi^{*}(s)$ lie on a single ray in the complex plane. This property is incompatible with (29) since $\lambda$ is not positive. The contradiction implies the validity of (iii). The proof of (iv) depends only on (iii) (see [8]). This completes the proof of Theorem 2'.

The content of the corollaries that follow summarize those applications of Theorem $2^{\prime}$ that we will require.

COROllaRy 1. Let $K(x, s)>0$ for $a<x, s<b$ and suppose there exists $u(x)>0(a<x<b)$ such that

$$
G(x, s)=\frac{K(x, s)}{u(x)}
$$

is continuous on $\Delta \times \Delta$ and positive for $(a \leqq x \leqq b, a<s<b)$; then (i)-(iv) of Theorem 2' hold for the operator $T$.

Corollary 2. Let $K(x, s)$ be $\operatorname{ETP}(x)\left(\right.$ see (19)); then (i)-(iv) of Theorem $2^{\prime}$ hold for the operator

$$
\left(T_{[p]} f\right)(\bar{x})=\int \dddot{\bar{\Delta}}_{-} \int K_{[p]}(\bar{x}, \bar{s}) f(\bar{s}) d \bar{s}, \quad \bar{x}, \bar{s} \in \Delta_{p} .
$$

Corollary 3. Suppose(31) is $\operatorname{ETP}(x)$; then (i)-(iv) of Theorem 2' hold for the operator (32).

In each case the desired conclusion cannot be obtained directly from Theorem 2 because of the possibly nonstrictness of the positivity of the kernel of the relevant operator.

3. Proof of Theorem 3. Before embarking on the proof of the main theorem, we review some terminology and preliminaries. The following formula is exceptionally useful in the subsequent analysis.

a. Let $X, Y$ and $Z$ represent subsets of the real line. If

$$
C(\xi, \zeta)=\int_{\mathbf{Y}} A(\xi, \eta) B(\eta, \zeta) d \sigma(\eta), \quad \xi \in X, \zeta \in Z,
$$

where $\sigma$ is a sigma finite regular measure and the integral converges absolutely, then

$$
C_{[p]}(\bar{\xi}, \bar{\zeta})=\int_{\Delta_{n}(Y)} \ldots \int A_{[p]}(\bar{\zeta}, \bar{\eta}) B_{[p]}(\bar{\eta}, \bar{\zeta}) d \sigma(\bar{\eta}), \quad \bar{\zeta} \in \Delta_{p}(X), \zeta \in \Delta_{p}(Z),
$$

where

$$
\Delta_{p}(X)=\left\{\xi=\left(\xi_{1}, \cdots, \xi_{p}\right) \mid \xi_{1}<\xi_{2}<\cdots<\xi_{p}, \xi_{i} \in X\right\}
$$

etc. 
The proof of (33) appears in [9, Problem 68, p. 48].

Let us introduce the following notation: if $f_{1}, f_{2}, \cdots, f_{p}$ are functions each defined on the interval $\bar{\Delta}_{1}=[a, b]$, we define their exterior or wedge product $f_{1} \wedge f_{2} \wedge \cdots \wedge f_{p}$ to be that function defined on the simplex $\Delta_{p}$ by

$$
\begin{aligned}
\left(f_{1} \wedge \cdots \wedge f_{p}\right)(\bar{x})=\operatorname{det}\left\|f_{i}\left(x_{j}\right)\right\|_{i, j=1}^{p}=F\left(\begin{array}{c}
1,2, \cdots, p \\
x_{1}, x_{2}, \cdots, x_{p}
\end{array}\right), \\
\bar{x}=\left(x_{1}, \cdots, x_{p}\right) \in \Delta_{p} .
\end{aligned}
$$

It is clear that (35) vanishes identically whenever any two component functions coincide (say $f_{i} \equiv f_{j}$ for some $i$ and $j(1 \leqq i<j \leqq p)$ ).

Now identifying $X=Y=[a, b], Z=$ the discrete finite set $\{1,2, \cdots, p\}$, $A(\xi, \eta)=K(\xi, \eta), B(\eta, \zeta)=f_{i}(\eta), C(\xi, \zeta)=\left(T f_{i}\right)(\xi)$ and applying (34) yields the important formula

$$
T f_{1} \wedge T f_{2} \wedge \cdots \wedge T f_{p}=T_{[p]}\left(f_{1} \wedge f_{2} \wedge \cdots \wedge f_{p}\right), \quad p=1,2, \cdots .
$$

With the formula (36) in hand, we are now prepared to present the proof of Theorem 3. For ease of exposition, the proof will be divided into a series of steps. For ready reference we repeat its statement here as given in the Introduction.

THEOREM 3. Let $K(x, y)$ be of class $C^{\infty}$ and ETP on $[a, b]$. Then the operator $T$ possesses a countable set of simple positive eigenvalues

$$
\lambda_{0}>\lambda_{1}>\lambda_{2}>\cdots>\lambda_{n}>\cdots
$$

decreasing to zero. There exists no other nonzero spectrum apart from the origin. Let $\phi_{0}(x), \phi_{1}(x), \phi_{2}(x), \cdots$ denote the corresponding eigenfunctions (each uniquely determined except for a multiplicative factor). Then

$$
\operatorname{sign} \varepsilon_{r} \Phi^{*}\left(\begin{array}{c}
0,1, \cdots, r \\
x_{1}, x_{2}, \cdots, x_{r}
\end{array}\right)>0 \quad\left(\bar{x} \in \bar{\Delta}_{r}\right)
$$

where $\varepsilon_{r}$ is appropriately +1 or -1 .

Let $\Lambda=\left\{\lambda_{0}, \lambda_{1}, \lambda_{2}, \cdots\right\}$ denote the nonzero eigenvalues of $T$ arranged so that

$$
r(T)=\left|\lambda_{0}\right| \geqq\left|\lambda_{1}\right| \geqq\left|\lambda_{2}\right| \geqq \cdots .
$$

The set $\Lambda$ may be empty, finite or countable. We recall (cf. Introduction) that the only possible limit point of $\Lambda$ is zero.

1. The hypothesis requires, in particular, that $K(x, s)>0$ on $\Delta \times \Delta$. Owing to Theorem 2, we conclude that $\lambda_{0}=r(T)>0$ is a simple eigenvalue, $\lambda_{0}>\left|\lambda_{i}\right|$, $i=1,2, \cdots$ provided any sush $\lambda_{i}$ exist and that the associated eigenfunction $\phi_{0}(x)$ is strictly of one sign. Thus (24) is verified for $r=0$.

We also know from (iv) of Theorem 2 that the Hilbert space $L_{2}(\Delta)$ can be decomposed as a direct sum

$$
L_{2}(\Delta)=\mathscr{M}\left(\lambda_{0}\right) \oplus \mathscr{U}_{0}\left(\lambda_{0}\right)
$$


where $\mathscr{M}\left(\lambda_{0}\right)$ is the characteristic manifold of the eigenspace belonging to $\lambda_{0}$, the linear space spanned by $\phi_{0}(x)$. Here $\mathscr{U}_{0}\left(\lambda_{0}\right)$ denotes the complementary space such that the operator $T$ restricted to $\mathscr{U}_{0}$ has a spectral radius $\left|\lambda_{1}\right|<\lambda_{0}$.

It is, a priori, conceivable that this spectral radius is zero (i.e., $\lambda_{1}$ does not exist or equals zero) and then $T$ is quasi nilpotent on $\mathscr{U}_{0}\left(\lambda_{0}\right)$. This will certainly happen if $\lambda_{1}$ does not exist. Actually in the course of our analysis we prove that $\left|\lambda_{1}\right|>0$.

2. Consider now the compound kernel of order 2

$$
K_{[2]}(\bar{x}, \bar{s}), \quad \bar{x} \in \Delta_{2}, \bar{s} \in \Delta_{2},
$$

and corresponding operator $T_{[2]}$. The hypotheses of the theorem plainly guarantee that $T_{[2]}$ is completely continuous and satisfy the conditions of Corollary 2. Appealing to Corollary 2 we conclude that $\gamma_{2}=r\left(T_{[2]}\right)>0$ is a simple eigenvalue and no other eigenvalue of $T_{[2]}$ exists with modulus $\gamma_{2}$. Moreover, part (iv) of Corollary 2 tells us that

$$
\frac{T_{[2]}^{n}}{\gamma_{2}^{n}}
$$

converges to a nontrivial projection operator on $\mathscr{M}^{[2]}\left(\gamma_{2}\right)$ (the characteristic manifold of $\gamma_{2}$ ).

We will now prove that $\lambda_{1}>0$ and $\gamma_{2}=\lambda_{0} \lambda_{1}$. Suppose first to the contrary that $\gamma_{2}>\lambda_{0}\left|\lambda_{1}\right|$. We write $f_{1}=a_{1} \phi_{0}+\psi_{1}$, permissible by (37) and $f_{2}=a_{2} \phi_{0}+\psi_{2}$ where $\psi_{i} \in \mathscr{U}_{0}\left(\lambda_{0}\right)(i=1,2)$. Then letting $\tilde{\gamma}_{2}=\gamma_{2} / \lambda_{0}>\left|\lambda_{1}\right|$ we obtain by (36) that

$$
\begin{aligned}
& \frac{T_{[2]}^{n}}{\gamma_{2}^{n}}\left(f_{1} \wedge f_{2}\right) \\
& \quad=a_{1}\left(\frac{T^{n} \phi_{0}}{\lambda_{0}^{n}} \wedge \frac{T^{n} \psi_{2}}{\tilde{\gamma}_{2}^{n}}\right)+a_{2}\left(\frac{T^{n} \psi_{2}}{\tilde{\gamma}_{2}^{n}} \wedge \frac{T^{n} \phi_{0}}{\lambda_{0}^{n}}\right)+\left(\frac{T^{n} \psi_{1}}{\lambda_{0}^{n}} \wedge \frac{T^{n} \psi_{2}}{\tilde{\gamma}_{2}^{n}}\right) .
\end{aligned}
$$

Now $T^{n} \phi / \lambda_{0}^{n}$ converges strongly for any $\phi$. In addition $T^{n} \psi / \tilde{\gamma}_{2}^{n} \rightarrow 0$ for all $\psi \in \mathscr{U}_{0}$ since the spectral radius of $T$ restricted to $\mathscr{U}_{0}$ is $\left|\lambda_{1}\right|<\tilde{\gamma}_{2}$.

Therefore $T_{[2]}^{n} / \gamma_{2}^{n}\left(f_{1} \wedge f_{2}\right) \rightarrow 0$ for any $f_{i} \in L_{2}\left(\Delta_{1}\right)(i=1,2)$. But the linear span of all functions of the form $f_{1} \wedge f_{2}$ are dense in $L_{2}\left(\Delta_{2}\right)$. It follows that the limit of the sequence of operators $T_{[2]}^{n} / \gamma_{2}^{n}$ must be zero in contradiction of (38). This shows that $0<\gamma_{2} \leqq \lambda_{0}\left|\lambda_{1}\right|$, and so $\left|\lambda_{1}\right|>0$. Let $\phi_{1}(x)$ be an eigenfunction associated with $\lambda_{1}$. Then (36) yields

$$
T_{[2]}\left(\phi_{0} \wedge \phi_{1}\right)=\lambda_{0} \lambda_{1}\left(\phi_{0} \wedge \phi_{1}\right)
$$

which shows that $\lambda_{0} \lambda_{1}$ is an eigenvalue of $T_{[2]}$ since $\phi_{1}$ and $\phi_{0}$ are linearly independent. By Corollary 2, part (iii) applied to $T_{[2]}$ we infer that $\lambda_{0} \lambda_{1}=r\left(T_{[2]}\right)=\gamma_{2}$ and therefore $\lambda_{1}>0$ and $\lambda_{1}>\left|\lambda_{i}\right|, i=2,3, \cdots$.

The proof of the last assertion goes as follows: 
Since $\lambda_{0} \lambda_{1}=\gamma_{2}=r\left(T_{[2]}\right)$ and $\lambda_{0} \lambda_{2}$ are eigenvalues of $T_{[2]}$ with eigenfunctions $\left(\phi_{0} \wedge \phi_{1}\right)$ and $\phi_{0} \wedge \phi_{2}$ respectively, we conclude on the basis of Corollary 2, part (iii)

$$
\lambda_{0} \lambda_{1}>\left|\lambda_{0} \lambda_{2}\right| \text { or } \lambda_{1}>\left|\lambda_{2}\right| \text {. }
$$

Moreover, again by virtue of Corollary 2, part (ii), we have that $\phi_{0} \wedge \phi_{1}$ is strictly of one sign on $\bar{\Delta}_{2}$ and this is (24) established now for the case $r=1$. The same corollary shows that $\lambda_{0} \lambda_{1}$ is a simple eigenvalue of $T_{[2]}$. It now follows that $\lambda_{1}$ is an eigenvalue of $T$ of geometric and algebraic multiplicity 1 . Indeed, let $\phi_{1}$ and $\phi_{1}^{*}$ be two eigenfunctions of $T$ for $\lambda_{1}$. Then $\phi_{0} \wedge \phi_{1}$ and $\phi_{0} \wedge \phi_{1}^{*}$ are eigen-functions of $T_{[2]}$ for $\lambda_{0} \lambda_{1}=\gamma_{2}$. But $\mathscr{M}_{[2]}\left(\gamma_{2}\right)$ is one-dimensional and so $\phi_{0} \wedge\left(\alpha \phi_{1}+\beta \phi_{1}^{*}\right) \equiv 0$ for some real constants $\alpha$ and $\beta$. But eigenfunctions $\phi_{0}$ and $\alpha \phi_{1}+\beta \phi_{1}^{*}$ belong to distinct eigenvalues ( $\lambda_{0}$ and $\lambda_{1}$ respectively) and therefore are independent; thus $\alpha \phi_{1}+\beta \phi_{1}^{*} \equiv 0$, i.e., the geometric multiplicity for $\lambda_{1}$ is 1 . Now suppose that $\left(T-\lambda_{1} I\right)^{2} \tilde{\phi}=0$. Since $\operatorname{dim} \mathscr{M}\left(\lambda_{1}\right)=1$, it follows that $T \tilde{\phi}-\lambda_{1} \tilde{\phi}=a \phi_{1}$ for some real $a$.

We form $\left(T_{[2]}-\gamma_{2} I_{[2]}\right)\left(\phi_{0} \wedge \tilde{\phi}\right)$ which by direct computation gives

$$
\left(T_{[2]}-\gamma_{2} I_{[2]}\right)\left(\phi_{0} \wedge \tilde{\phi}\right)=a \lambda_{0}\left(\phi_{0} \wedge \phi_{1}\right)
$$

and so

$$
\left(T_{[2]}-\gamma_{2} I_{[2]}\right)^{2}\left(\phi_{0} \wedge \tilde{\phi}\right)=0 .
$$

But the algebraic multiplicity of $T_{[2]}$ for $\gamma_{2}$ is 1 . The relations (41) and (42) are consistent with this fact only if $a=0$. This shows that $\left(T-\lambda_{1} I\right)^{2} \tilde{\phi}=0$ implies $\left(T-\lambda_{1} I\right) \tilde{\phi}=0$ which means that $\lambda_{1}$ has simple algebraic multiplicity.

With the existence of $\lambda_{0}$ and $\lambda_{1}$ in hand and their respective properties, we may rewrite the decomposition (37) as a direct sum

$$
L_{2}(I)=\mathscr{M}\left(\lambda_{0}\right) \oplus \mathscr{M}\left(\lambda_{1}\right) \oplus \mathscr{U}_{1}
$$

such that $\mathscr{M}\left(\lambda_{i}\right)=$ the characteristic manifold of $\lambda_{i}(i=0,1)$ each of which is one-dimensional and $T$ restricted to $\mathscr{U}_{1}$ has spectral radius $\left|\lambda_{2}\right|$. (Once more we point out that if $\lambda_{2}$ does not exist then $T$ restricted to $\mathscr{U}_{1}$ is quasi nilpotent. However, we will, in fact, prove below in paragraph 3 that $\left|\lambda_{2}\right|>0$.)

3. Consider now the compound operator of order $3, T_{[3]}$ whose corresponding kernel is

$$
K_{[3]}(\bar{x}, \bar{s}), \quad \bar{x} \in \Delta_{3}, \bar{s} \in \Delta_{3} .
$$

Trivially, $T_{[3]}$ is completely continuous. By Corollary 2, we have that $\gamma_{3}=r\left(T_{[3]}\right)>0$ is a simple eigenvalue of $T_{[3]}$. The remaining spectrum of $T_{[3]}$ is contained in the circle $|\lambda|<\gamma_{3}$. We also know on the basis of Corollary 2, part (iv) that

$$
\frac{T_{[3]}^{n}}{\gamma_{3}^{n}}
$$


converges to a nonzero projection operator on the one-dimensional manifold $\mathscr{M}^{13]}\left(\gamma_{3}\right)$. We now prove that $\gamma_{3}=\lambda_{0} \lambda_{1}\left|\lambda_{2}\right|$. To this end, suppose first to the contrary that $\gamma_{3}>\lambda_{0} \lambda_{1}\left|\lambda_{2}\right|$ and set $\tilde{\gamma}_{3}=\gamma_{3} / \lambda_{0} \lambda_{1}$. Then $\tilde{\gamma}_{3}>\left|\lambda_{2}\right|$. By the same methods as in (39), we deduce that

$$
\frac{T_{[3]}^{n}}{\gamma_{3}^{n}}\left(\phi_{0} \wedge \phi_{1} \wedge \psi\right)=\left(\frac{T^{n} \phi_{0}}{\lambda_{0}^{n}} \wedge \frac{T^{n} \phi_{1}}{\lambda_{1}^{n}} \wedge \frac{T^{n} \psi}{\tilde{\gamma}_{3}^{n}}\right)
$$

tends to zero for $\psi \in \mathscr{U}_{2}$, and similarly

and

$$
\frac{T_{[3]}^{n}}{\gamma_{3}^{n}}\left(\phi_{0} \wedge \psi_{1} \wedge \psi_{2}\right), \frac{T_{[3]}^{n}}{\gamma_{3}^{n}}\left(\psi_{1} \wedge \phi_{1} \wedge \psi_{2}\right), \quad \psi_{i} \in \mathscr{U}_{2},
$$

$$
\frac{T_{[3]}^{n}}{\gamma_{3}^{n}}\left(\psi_{1} \wedge \psi_{2} \wedge \psi_{3}\right)
$$

all converge strongly to zero as $n \rightarrow \infty$. Using (43) as we have previously used (37), this implies that $T_{[3]}^{n} / \gamma_{3}^{n}$ converges to the zero operator contradicting (44). It follows that $\gamma_{3} \leqq \lambda_{0} \lambda_{1}\left|\lambda_{2}\right|$ and so $\left|\lambda_{2}\right|>0$. The existence of a nontrivial eigenfunction $\phi_{2}(x)$ corresponding to $\lambda_{2}$ is now assured. Thus, we write $T \phi_{2}=\lambda_{2} \phi_{2}$. Referring to (36) we see that $\phi_{0} \wedge \phi_{1} \wedge \phi_{2}$ is an eigenfunction of $T_{[3]}$ with eigenvalue $\lambda_{0} \lambda_{1} \lambda_{2}$. Applying Corollary 2, we may conclude that $\gamma_{3}=\lambda_{0} \lambda_{1} \lambda_{2}$ (i.e., $\lambda_{2}>0$ ) and $\phi_{0} \wedge \phi_{1} \wedge \phi_{2}$ is the unique (apart from a multiplicative constant) eigenfunction associated with $\gamma_{3}$. Moreover, $\phi_{0} \wedge \phi_{1} \wedge \phi_{2}$ maintains a strict sign for $\bar{x} \in \bar{\Delta}_{3}$. The proof that $\lambda_{2}>\left|\lambda_{3}\right|$ is done as before.

The proof that $\operatorname{dim} \mathscr{M}\left(\lambda_{2}\right)=1$ is identical to the proof that $\operatorname{dim} \mathscr{M}\left(\lambda_{1}\right)=1$. To show that $\lambda_{2}$ is of algebraic multiplicity 1 , now suppose $\left(T-\lambda_{2} I\right)^{2} \tilde{\phi}=0$. This implies $T \tilde{\phi}=\lambda_{2} \tilde{\phi}+a \phi_{2}$ for some real $a$. A direct computation gives

$$
\left(T_{[3]}-\gamma_{3} I_{[3]}\right)\left(\phi_{0} \wedge \phi_{1} \wedge \tilde{\phi}\right)=a \lambda_{0} \lambda_{1} \phi_{0} \wedge \phi_{1} \wedge \phi_{2}
$$

and this is only possible if $a=0$. Thus $\lambda_{2}$ is of algebraic multiplicity 1 . In summary, the preceding analysis has established (24) for the case $r=2$.

The pattern of the proof is now clear. The general step is accomplished with the aid of an induction procedure. Actually, the arguments for $T_{[3]}$ embodies virtually all the general reasoning with trivial adjustments. The proof is therefore complete.

By referring appropriately to Corollary 3 in place of Corollary 2 for the preceding analysis, we may extend the assertion of Theorem 3 to the following situation.

Corollary 4. Let $K(x, s)$ be continuous and suppose

$$
L_{p}(\bar{x}, \bar{s})=\frac{K\left(\begin{array}{c}
x_{1}, \cdots, x_{p} \\
s_{1}, \cdots, s_{p}
\end{array}\right)}{q(\bar{x})}
$$

is continuous and strictly positive on the set $\Delta_{p} \times \Delta_{p}$ where 


$$
q(\bar{x})=\left[\prod_{p \geqq j>i \geqq 1}\left(x_{j}-x_{i}\right)\right] \prod_{i=1}^{p}\left(x_{i}-a\right)\left(b-x_{i}\right) \quad(p=1,2, \cdots) .
$$

Moreover, assume that $L_{p}(\bar{x}, \bar{s})$ can be extended to the closure $\bar{\Delta}_{p} \times \bar{\Delta}_{p}$ so that it remains continuous and strictly positive $(r=1,2, \cdots)$.

Then the nonzero values of the spectrum of the operator $T$ consists of a countable number of simple positive eigenvalues

$$
\lambda_{0}>\lambda_{1}>\cdots>\lambda_{n}>\cdots
$$

decreasing to zero. The corresponding eigenfunctions satisfy

$$
\operatorname{sign} \varepsilon_{p} \Phi^{*}\left(\begin{array}{c}
0,1, \cdots, p \\
x_{1}, x_{2}, \cdots, x_{p}
\end{array}\right)>0 \quad(p=1,2,3, \cdots)
$$

for all

$$
a<x_{1} \leqq x_{2} \leqq \cdots \leqq x_{p}<b .
$$

It is worthwhile to emphasize that under the present set of hypotheses, strict inequality in (48) need not hold when $x_{1}=a$ and/or $x_{p}=b$ as contrasted with assertion (24).

4. Extensions.

A. The result of Theorem 3 can be generalized. To this end, we merely require that $K(x, s)$ is continuous, (8) is satisfied and for each $k$ there exists some iterate, say the $r_{k}$ th such that $K^{r_{k}}(x, s)$ is $\operatorname{ETP}(x)$ or order $k$. The last condition means that (19) is assumed to hold only for $p \leqq k$.

Let us examine the validity of Theorem 3 under these hypothesis. Let $\Gamma=\left\{\lambda_{i}\right\}_{i=0}^{\infty}$ denote such eigenvalues of the operator $T$ of (1), as may exist arranged in decreasing order of magnitude, i.e.,

$$
\left|\lambda_{0}\right| \geqq\left|\lambda_{1}\right| \geqq\left|\lambda_{2}\right| \geqq \cdots \text {. }
$$

Each eigenvalue is to be repeated as frequently as its geometric multiplicity.

Let $\left\{\phi_{i}(x)\right\}_{i=0}^{\infty}$ represent the related eigenfunctions. We do not know of the existence of any of the $\lambda_{i}$ as yet. Our aim is to establish their existence and elaborate the properties of the corresponding eigenfunctions.

Let $r_{1}$ be such that $K^{\left(r_{1}\right)}(x, s)>0$. The spectrum of $T^{r_{1}}$ consists of the set $\left\{\lambda_{i}^{r_{k}}\right\}_{i=0}^{\infty}$ with the same set of eigenfunctions $\left\{\phi_{i}(x)\right\}_{i=0}^{\infty}$. According to Jentzsch's theorem with strictly positive kernel, we have

$$
\lambda_{0}^{r_{1}}>0
$$

and the existence of a corresponding eigenfunction $\phi_{0}(x)$ which is strictly positive on $[a, b]$. Moreover $\lambda_{0}^{r_{1}}$ exceeds in absolute value every other eigenvalue of $T^{r_{1}}$. The inequality implies that the spectral radius of $T$ is positive and equal to $\left|\lambda_{0}\right|$. By virtue of the Theorem 1 applied to non-negative kernels we know that $\left|\lambda_{0}\right|$ is 
an eigenvalue of $T$ possessing an associated non-null non-negative eigenfunction $\phi_{0}(x)$. But $\lambda_{0}^{r_{1}}=\left|\lambda_{0}\right|^{r}>0$ is a simple eigenvalue for $T^{r_{1}}$ and therefore $\lambda_{0}=\left|\lambda_{0}\right|$ is a simple eigenvalue for $T$ whose eigenmanifold is spanned by $\phi_{0}(x)$. We also conclude due to the property $K^{\left(r_{1}\right)}(x, s)>0$ that $\lambda_{0}$ is a simple eigenvalue for $T^{*}$ whose eigenfunction $\psi_{0}$ is strictly of one sign. It then follows that $\lambda_{0}$ is of simple algebraic multiplicity.

By virtue of the simple nature of the eigenvalue $\lambda_{0}$ and since manifestly $\lambda_{0}>\left|\lambda_{t}\right|, i=1,2, \cdots$, we obtain a decomposition of $L_{2}$ as a direct sum $L_{2}=\mathscr{M}\left(\phi_{0}\right) \oplus \mathscr{E}\left(\phi_{0}\right)$ where $T$ restricted to $\mathscr{E}\left(\phi_{0}\right)$ has spectral radius $\left|\lambda_{1}\right|$ and $\mathscr{M}\left(\phi_{0}\right)$ is the eigenmanifold spanned by $\phi_{0}$.

Next passing to the compound operator of order 2, we have by the assumption that

$$
\frac{K_{[2]}^{\left(r_{2}\right)}(\bar{x}, \bar{s})}{u(x)} \quad\left(\bar{x}, \bar{s} \in \Delta_{2} ; u(x)=x_{2}-x_{1}\right)
$$

is continuous and strictly positive for $\bar{x} \in \bar{\Delta}_{2}$ and $\bar{s} \in \Delta_{2}$.

Arguing in terms of the operators $T^{r_{2}}$ and its iterates we deduce exactly as in Theorem 3, the existence of $\left|\lambda_{1}\right|>0$ such that

$$
\lambda_{0}^{r_{2}} \lambda_{1}^{r_{2}}>0
$$

and $\lambda_{0}^{r_{2}} \lambda_{1}^{r_{2}}$ is the spectral radius of $T_{[2]}^{r_{2}}$. This means that the spectral radius of $T_{[2]}$ is nonzero and since $T_{[2]} \geqq 0$, Theorem 1 tells us that $\lambda_{0} \lambda_{1}>0$ is an eigenvalue for $T_{[2]}$ and therefore $\lambda_{1}=\left|\lambda_{1}\right|>0$. Since $\lambda_{0}^{r_{2}} \lambda_{1}^{r_{2}}$ has simple algebraically and geometrically multiplicity the same follows for $\lambda_{0} \lambda_{1}$ and consequently the same for $\lambda_{1}$. Continuing in this way, we deduce the results of Theorem 3 , under the conditions as stated at the start of this section. Also recognizing that the eigenfunction set is unchanged under the iteration of the operator, i.e., $T, T^{r}$ have the same set of eigenfunctions, we obtain

THEOREM 4. Let $K(x, s)$ be continuous on $\Delta \times \Delta$ and assume that (8) holds. Suppose that for each integer $k>1$ there exists an integer $r_{k} \geqq 1$ with the property that $K^{\left(r_{k}\right)}(x, s)$ is $\operatorname{ETP}(x)$ or order $k$ with respect to $a \leqq x \leqq b, a<s<b$. Under these conditions the conclusions of Theorem 3 hold.

B. The result of Theorem 4 can be extended in certain situations even when $K(a, s) \equiv 0$ and/or $K(b, s) \equiv 0$. Assume that (8) holds. Also, suppose that there exists a function $u(x)$, strictly positive in $(a, b)$ and vanishing appropriately at the endpoints and that for each integer $k$ there exists an integer $r_{k}$ such that

$$
\frac{K_{[k]}^{\left(r_{k}\right)}(\bar{x}, \bar{s})}{\prod_{k \geqq j>i \geqq 1}\left(x_{j}-x_{i}\right) \prod_{i=1}^{k} u\left(x_{i}\right)}
$$


is continuous and strictly positive on $\bar{\Delta}_{k} \times \Delta_{k}$. The function $u(x)$ is here playing the role of $\prod\left(x_{j}-x_{i}\right)$ for that part of the boundary of $\bar{\Delta}_{k}$ at which $x_{1}=a$ and/or $x_{k}=b$.

Virtually duplicating the proof of Theorem 4, we obtain

THEOREM 5. Under the condition expressed in (52), the operator $T$, induced by the kernel $K(x, s)$, possesses a countable set of positive eigenvalues

$$
\lambda_{0}>\lambda_{1}>\lambda_{2}>\cdots>0
$$

decreasing to zero. Each eigenvalue has simple algebraic and geometric multiplicity. Let $\phi_{0}(x), \phi_{1}(x), \phi_{2}(x), \cdots$, denote respective eigenfunctions and let

$$
\tilde{\phi}_{i}(x)=\frac{\phi_{i}(x)}{u(x)}, \quad i=0,1,2, \cdots
$$

Then

$$
\varepsilon_{p} \tilde{\Phi}\left(\begin{array}{c}
0,1,2, \cdots, p-1 \\
x_{1}, x_{2}, \cdots, x_{p}
\end{array}\right)>0 \text { for all } \bar{x} \in \bar{\Delta}_{p}
$$

Actually, the functions $\tilde{\phi}_{i}(x)(i=0,1,2, \cdots)$ are the eigenfunctions of the integral operator whose kernel is

$$
M(x, s)=\frac{K(x, s)}{u(x)} u(s)
$$

This function has the property that for each integer $k>0$ there exists an iterate $\boldsymbol{r}_{\boldsymbol{k}}$ such that $M^{\left(r_{k}\right)}(x, s)$ is ETP for $a \leqq x \leqq b, a<s<b$ and Theorem 4 applies.

We close this paper by reviewing some of the more refined oscillation structure of the eigenfunctions $\left\{\phi_{n}(x)\right\}_{n=0}^{\infty}$ valid under the hypothesis of Theorem 3. For example, it is easy to prove that (24) implies that

$$
\phi(x)=\sum_{i=p}^{q} c_{i} \phi_{i}(x), \quad c_{i} \text { real, } \sum c_{i}^{2}>0
$$

has the property

$$
p \leqq S(\phi(x)) \leqq Z(\phi(x)) \leqq q
$$

where $Z(\phi)$ denotes the number of zeros of $\phi$ counting multiplicities and $S(\phi)$ represents the number of variation of sign of the function $\phi(x)$ as $x$ traverses $[a, b]$. Two important consequences of (53) are that

$$
S\left(\phi_{i}(x)\right)=Z\left(\phi_{i}(x)\right)=i
$$

and the property that the zeros of $\phi_{i}(x)$ and $\phi_{i+1}(x)$ strictly interlace.

The assertions (53) and (54) are essentially contained in Gantmacher and Krein [1] in a context in which the existence of eigenfunctions is assured by the assumption that $K(x, s)$ is symmetric. 
Let $D(x, y, \lambda)$ denote the resolvent kernel

$$
D(x, y, \lambda)=K(x, s)+\sum_{n=1}^{\infty} \frac{(-\lambda)^{n}}{n !} \int_{a}^{b} \ldots \int_{a}^{b} K\left(\begin{array}{c}
x, s_{1} \cdots s_{n} \\
s, s_{1} \cdots s_{n}
\end{array}\right) d s_{1} d s_{2} \cdots d s_{n} .
$$

Under the conditions of Theorem 3 we can prove that

$$
U(x)=\alpha D(x, a, \lambda)+\beta D(x, b, \lambda) \quad\left(\alpha, \beta \text { real, } \alpha^{2}+\beta^{2}>0\right)
$$

satisfies

$$
k-1 \leqq S(U(x))=Z(U(x)) \leqq k+2
$$

for $\lambda$ located in the interval $\lambda_{k}<\lambda<\lambda_{k+1}$ where $\lambda_{0}, \lambda_{1}, \lambda_{2}, \cdots$ denote the eigenvalues of the integral operator (1).

The zeros of $U(x)$ and $V(x)=\gamma D(x, a, \lambda)+\delta D(x, b, \lambda)$ where $\gamma, \delta$ are real and satisfy $\alpha \delta-\beta \gamma \neq 0$ strictly interlace. Finally the resolvent kernel $D(x, y, \lambda)$ is related to the eigenvectors by the formula

$$
D\left(x, a, \lambda_{k}\right) \equiv c_{k} D\left(x, b, \lambda_{k}\right) \equiv d_{k} \phi_{k}(x)
$$

where $c_{k}$ and $d_{k}$ are appropriate constants.

These last results and other refinements and extensions will be elaborated elsewhere.

\section{REFERENCES}

1. F. Gantmacher and M. Krein, Oscillation matrices and kernels and small oscillations of mechanical systems, 2nd ed., Gosudarstv. Izdat. Tehn.-Teor. Lit., Moscow, 1950. (Russian)

2. S. Karlin, Oscillation properties of eigenfunctions of totally positive integral operators, (to appear).

3. - Total positivity and convexity preserving transformations, Proc. Sympos. Pure Math. Vol. 7, pp. 329-347, Amer. Math. Soc., Providence, R.I., 1963. appear).

4. —_ Total positivity and applications, Stanford Univ. Press, Stanford, Calif. (to

5. M. G. Krein and M. A. Rutman, Linear operators leaving invariant a cone in a Banach space, Uspehi Mat. Nauk 3 (1948), 3-95; Amer. Math. Soc. Transl. no. 26, 1950; reprint, Amer. Math. Soc. Transl. (1) 10 (1962), 199-325.

6. G. Birkhoff, Extensions of Jentzsch's theorem, Trans. Amer. Math. Soc. 85 (1957), 219-228.

7. S. Karlin, Positive operator, J. Math. Mech. 8 (1959), 907-938.

8. K. Yosida and S. Kakutani, Operator-theoretical treatment of Markoff's process and mean ergodic theorem, Ann. of Math. (2) 42 (1951), 188-228.

9. G. Pólya and G. Szegö, Aufgaben and Lehrsatze aus der Analysis, Vol. II, Springer, Berlin, 1925.

\section{STANFORD UNIVERSITY,}

Stanford, CaLIfornia 